\title{
Dopaminergic Response to Drug Words in Cocaine Addiction
}

\author{
Rita Z. Goldstein, ${ }^{1}$ Dardo Tomasi, ${ }^{1}$ Nelly Alia-Klein, ${ }^{1}$ Jean Honorio Carrillo,,${ }^{1,2}$ Thomas Maloney, ${ }^{1}$ Patricia A. Woicik, ${ }^{1}$ \\ Ruiliang Wang, ${ }^{1}$ Frank Telang, ${ }^{1,3}$ and Nora D. Volkow ${ }^{3,4}$ \\ ${ }^{1}$ Department of Medical Research, Center for Translational Neuroimaging, Brookhaven National Laboratory, Upton, New York 11973-5000, ${ }^{2}$ State \\ University of New York at Stony Brook, Stony Brook, New York 11794-4400, ${ }^{3}$ National Institute on Alcohol Abuse and Alcoholism, Rockville, Maryland \\ 20857, and ${ }^{4}$ National Institute on Drug Abuse, Bethesda, Maryland 20892
}

When exposed to drug conditioned cues (stimuli associated with the drug), addicted individuals experience an intense desire for the drug, which is associated with increased dopamine cell firing. We hypothesized that drug-related words can trigger activation in the mesencephalon, where dopaminergic cells are located. During functional magnetic resonance imaging (fMRI), 15 individuals with cocaine use disorders and 15 demographically matched healthy control subjects pressed buttons for color of drug-related versus neutral words. Results showed that the drug words, but not neutral words, activated the mesencephalon in the cocaine users only. Further, in the cocaine users only, these increased drug-related mesencephalic responses were associated with enhanced verbal fluency specifically for drug words. Our results for the first time demonstrate fMRI response to drug words in cocaine-addicted individuals in mesencephalic regions as possibly associated with dopaminergic mechanisms and with conditioning to language (in this case drug words). The correlation between the brief verbal fluency test, which can be easily administered (crucial for clinical studies), and fMRI cue reactivity could be used as a biomarker of neurobiological changes in addiction.

\section{Introduction}

The mesencephalon (location of the ventral tegmental area and substantia nigra) is a major source of dopaminergic release in response to motivationally salient or conditioned stimuli (Robinson and Berridge, 1993; McClure et al., 2003). Possibly as a result of supraphysiological and chronic reactions to drugs of abuse, a marked decrease in baseline (tonic) dopamine receptor availability and in dopamine release has been reliably documented in drug-addicted individuals (Volkow et al., 2004). When exposed to drug conditioned cues (stimuli associated with the drug), however, addicted individuals experience an intense desire for the drug as associated with an increase in dopamine cell firing (Volkow et al., 2006), supporting a crucial role for the mesencephalon in cue-induced relapse to drug seeking (Bossert et al., 2004). The goal of the current study was to investigate the role of verbal descriptors of drug stimuli (words), which have been conditioned to drug use in humans, in evoking similar dopaminergic responses in addicted individuals as estimated with novel behavioral and functional magnetic resonance imaging (fMRI) tasks. We hypothesized that drug but not neutral words would be asso-

Received Sept. 5, 2008; revised Feb. 13, 2009; accepted March 19, 2009.

This study was supported by grants from the National Institute on Drug Abuse (Grants 1R01DA023579 and R21DA02062 to R.Z.G.) and the General Clinical Research Center (Grant 5-M01-RR-10710). We thank Muhammad A Parvaz for help with task administration, Alex Panagopoulos and Dimitris Samaras for help with early data analyses, and Gene-Jack Wang for help with medical screens. We also thank SahibS. Khalsa and Steve Berry for help with word selection, matching, and ratings and Suparna Rajaram for help with early task design.

Correspondence and requests for materials should be addressed to Dr. Rita Z. Goldstein, Department of Medical Research, Center for Translational Neuroimaging, Brookhaven National Laboratory, P. 0. Box 5000, Upton, NY 11973-5000. E-mail: rgoldstein@bnl.gov.

DOI:10.1523/JNEUROSCI.4247-08.2009

Copyright (C) 2009 Society for Neuroscience $\quad$ 0270-6474/09/296001-06\$15.00/0 ciated with enhanced cue reactivity as measured at both the behavioral (assessed by correct verbal output on a neuropsychological semantic fluency test) and mesencephalon response levels, and that these brain-behavior responses would be intercorrelated in cocaine-addicted individuals but not in demographically matched healthy control participants.

\section{Materials and Methods}

Participants. A total of 30 right-handed native English speakers, 15 individuals with current cocaine use disorders (CUDs) and 15 healthy control participants, were recruited using advertisements in local newspapers and by word of mouth. All subjects were able to understand and give informed consent (for inclusion/exclusion criteria, see supplemental material, available at www.jneurosci.org). Healthy controls and CUDs were matched on sex, age, education, and general intellectual functioning (Table 1).

The CUDs were not currently seeking treatment as ascertained during an extensive psychiatric screen that included the Structured Clinical Interview for DSM-IV Axis I Disorders [research version (First et al., 1996; Ventura et al., 1998)] and the Addiction Severity Index (McLellan et al., 1992). Indeed all CUDs used crack/cocaine (mostly via the smoked route) in the past $30 \mathrm{~d}$ and met DSM-IV criteria for current cocaine dependence $(N=12)$ or abuse $(N=3$; these three subjects met criteria for cocaine dependence in remission). One CUD also reported current alcohol abuse. Current use or dependence on other drugs was denied and corroborated by prescan urine tests in all subjects (urine was positive for cocaine in 10 CUDs; urine was negative for all drugs in all other subjects). Subjects were fully informed of all study procedures and risks associated with MRI, providing written consent for their involvement in this study in accordance with the local Institutional Review Board.

The drug word fMRI task. All participants were scanned during a drug word fMRI task while viewing, in a blocked manner, drug or neutral 
Table 1. Demographics and drug use of all study subjects

\begin{tabular}{|c|c|c|c|}
\hline & $\begin{array}{l}\text { Test }^{a} \\
\chi_{1}^{2}, Z, \text { or } t_{28}\end{array}$ & $\begin{array}{l}\text { Control } \\
N=15\end{array}$ & $\begin{array}{l}\text { Cocaine } \\
N=15\end{array}$ \\
\hline Gender: Male/female & 0.7 & $10 / 5$ & $12 / 3$ \\
\hline Race: African-American/Caucasian/Hispanic/Asian & $7.6_{3}$ & $8 / 5 / 1 / 1$ & $14 / 0 / 1 / 0$ \\
\hline Laterality quotient (Oldfield, 1971) & $-0.3^{3}$ & $0.92 \pm 0.03$ & $0.91 \pm 0.03$ \\
\hline Age (years) & 1.7 & $39.6 \pm 1.8$ & $43.6 \pm 1.4$ \\
\hline Education (years) & -1.3 & $14.5 \pm 0.6$ & $13.4 \pm 0.5$ \\
\hline Verbal IQ: Wide Range Achievement Test III, Reading scale (Wilkinson, 1993) & -0.6 & $102.5 \pm 2.4$ & $100.5 \pm 2.6$ \\
\hline Nonverbal IQ: WASI, Matrix reasoning scale (Wechsler, 1999) & -0.1 & $11.0 \pm 0.8$ & $10.9 \pm 0.5$ \\
\hline Depression: Beck Depression Inventory II (Beck et al., 1996) & -0.3 & $3.3 \pm 1.0$ & $4.4 \pm 1.4$ \\
\hline Socioeconomic status: Hollingshead index & -1.2 & $38.0 \pm 3.7$ & $32.3 \pm 3.0$ \\
\hline Cigarette smokers (current or past/nonsmokers) & $8.6^{*}$ & $4 / 11$ & $12 / 3$ \\
\hline Daily cigarettes (current smokers: $\mathrm{N}=3 / 11$ ) & -0.2 & $10.0 \pm 4.0$ & $9.6 \pm 1.9$ \\
\hline Time since last cigarette (within $4 \mathrm{~h} />4 \mathrm{~h} /$ overnight or more) & $1.1_{2}$ & $1 / 2 / 0$ & $5 / 4 / 2$ \\
\hline Age of onset of cocaine use (years) & & & $24.9 \pm 1.6$ \\
\hline Duration of cocaine use (years) & & & $15.7 \pm 1.1$ \\
\hline Days/week of cocaine use during the last $30 \mathrm{~d}$ & & & $3.6 \pm 0.6$ \\
\hline Duration of current abstinence (days) & & & $5.1 \pm 1.1$ \\
\hline Withdrawal symptoms: 18-item CSSA (0-126) (Kampman et al., 1998) & & & $11.0 \pm 2.0$ \\
\hline Cocaine craving: 5 -item questionnaire (0 - 45) (Tiffany et al., 1993) & & & $16.3 \pm 2.3$ \\
\hline
\end{tabular}

Values are frequencies or means \pm SEM (unless otherwise indicated). WASI, Wechsler Abbreviated Scale of Intelligence; CSSA, Cocaine Selective Severity Assessment Scale.

${ }^{a} \chi^{2}$ tests were used for categorical variables, Mann-Whitney $U$ for continuous non-normally distributed variables, and $t$ test for normally distributed continuous variables.

${ }^{*} p<0.01$.

words (2000 ms per word). Specifically, this fMRI task (developed in E-prime, Psychology Software Tools) uses 40 drug words matched with 40 household words on length, part of speech (noun, adjective, adverb, verb), and frequency in the English language [here we referred to the most widely used dictionary designed specifically for this purpose (Francis and Kučera, 1982): the mean frequency for the drug words was 43.7 and that of the neutral words was 42.6] (Goldstein et al., 2007b). We did not include non-English or slang drug words that may not have been recognized by the control subjects. Similar to other emotional fMRI tasks (Whalen et al., 1998), the two word types were presented via MRIcompatible goggles in a blocked on-off or off-on order (i.e., drugneutral or neutral-drug), counterbalanced between subjects. There were eight 3.4 min task repetitions (four drug, four neutral), each containing two blocks of 20 drug or neutral words, interleaved with a $20 \mathrm{~s}$ fixation cross. Each word trial was composed of a $500 \mathrm{~ms}$ fixation cross, a $2000 \mathrm{~ms}$ word presentation (for word reading), a $500 \mathrm{~ms}$ response window, and a $500 \mathrm{~ms}$ feedback slide. During the $500 \mathrm{~ms}$ response window, subjects had to press one of four buttons (yellow, blue, red, green) on a commercially available MRI response pad (Cedrus brand Lumina model LP-400), matching the color of the word they had just read; word color order was pseudorandomized across all task runs. Note that the overt behavioral Stroop effect was not expected because, to encourage processing of the meaning of the word and minimize the expected working-memory difficulties in CUDs (Woicik et al., 2009), we separated word reading from pressing for its color (by $2000 \mathrm{~ms}$ ), thereby decreasing the conflict inherent in such a task (as further described in supplemental material, available at www.jneurosci.org). Last, each word sequence was performed under one of four counterbalanced monetary reward amounts $(50 \$, 25 \$$, $1 \$$, or $0 \$$ ), gained for correct performance for up to $\$ 75$ received at the completion of this study ( $\$ 66.6 \pm 1.1$ with no differences between the groups in this amount, $\left.t_{(28)}=0.5, p>0.7\right)$.

The word fluency task. All subjects also performed a standard semantic fluency task, naming as many words from two specified semantic categories (animals and fruits or vegetables) for $1 \mathrm{~min}$ per category (Lezak, 1995). Subjects also participated in the emotional variant of this task, naming drug-related words (names of drugs, people, places, or states of mind related to getting, using, or recovering from drugs) for the same duration (Goldstein et al., 2007c). Correct responses (excluding repetitions and errors, i.e., words clearly not related to the selected semantic category) were summed for each category. After the fMRI measures, we calculated a differential drug $>$ neutral (averaged across both non-drug semantic categories) fluency measure; here we first corrected, with regression analyses, for verbal IQ (intelligence quotient) that correlated with the fluency measures in the CUDs (supplemental material, available at www.jneurosci.org). The unstandardized residuals were used for all subsequent analyses.

MRI data acquisition. MRI scanning was performed on a $4 \mathrm{~T}$ wholebody Varian/Siemens MRI scanner. The blood oxygenation leveldependent (BOLD) responses were measured as a function of time using a $\mathrm{T}_{2}{ }^{*}$-weighted single-shot gradient-echo echoplanar imaging sequence [echo time/repetition time $=20 / 1600 \mathrm{~ms}, 4 \mathrm{~mm}$ slice thickness, $1 \mathrm{~mm}$ gap, typically 33 coronal slices, $20 \mathrm{~cm}$ field of view, $64 \times 64$ matrix size, $90^{\circ}$ flip angle, $200 \mathrm{kHz}$ bandwidth with ramp sampling, 128 time points, and 4 dummy scans to be discarded to avoid nonequilibrium effects in the fMRI signal). Padding was used to minimize motion, which was also monitored immediately after each fMRI run (Caparelli et al., 2003). Earplugs and headphones were used to minimize the interference effect of scanner noise during fMRI (Tomasi et al., 2005). Anatomical images were collected using a $\mathrm{T}_{1}$-weighted 3D-MDEFT (three-dimensional modified driven equilibrium Fourier transform) sequence (Lee et al., 1995) and a modified $\mathrm{T}_{2}$-weighted hyperecho sequence (Hennig and Scheffler, 2001), and were reviewed to rule out gross brain morphological abnormalities.

MRI data processing. Subsequent analyses were performed with the statistical parametric mapping package (SPM2) (Wellcome Trust Centre for Neuroimaging, London, UK). A six-parameter rigid body transformation ( 3 rotations, 3 translations) was used for image realignment and to correct for head motion; $2 \mathrm{~mm}$ displacement and $2^{\circ}$ rotation in any of the axes in any of the task repetitions were used as criteria for acceptable motion. The realigned datasets were spatially normalized to the standard frame (Talairach) with a 12-parameter affine transformation (Ashburner et al., 1997), using a voxel size of $3 \times 3 \times 3 \mathrm{~mm}$. An $8 \mathrm{~mm}$ full-widthhalf-maximum Gaussian kernel was used to smooth the data.

BOLD-fMRI analyses. A general linear model (Friston et al., 1995) and a box-car design convolved with a canonical hemodynamic response function and high-pass filter (cutoff frequency, 1/520 s) was used to calculate individual BOLD-fMRI maps for the fMRI task. Two contrasts per subject, reflecting percentage signal change for the drug or neutral words from the fixation baseline, were used to calculate the direct drug $>$ neutral differential contrast of interest, which was used in all subsequent analyses in SPM2: two one-way $t$ tests (drug $>$ neutral comparison separately for CUDs and controls), a two-way $t$ test (direct group comparison, CUDs vs controls, for the drug $>$ neutral contrast), and simple regression analyses (using the drug $>$ neutral contrast maps) with selected variables as seed values (drug $>$ neutral word fluency or mesencephalon drug $>$ neutral \% BOLD signal change for every participant) 


\begin{tabular}{|c|c|c|c|c|c|c|}
\hline \multirow[b]{2}{*}{ Region of activation and variable } & \multirow[b]{2}{*}{ Laterality } & \multicolumn{3}{|c|}{ Talairach coordinates } & \multirow[b]{2}{*}{ Voxels } & \multirow[b]{2}{*}{$t$-stat ${ }^{a}$} \\
\hline & & $x$ & $y$ & $z$ & & \\
\hline \multicolumn{7}{|l|}{ Cocaine-addicted individuals } \\
\hline \multicolumn{7}{|l|}{ Drug $>$ neutral words } \\
\hline Mesencephalon (substantia nigra) & $\mathrm{R}$ & 6 & -12 & -9 & 39 & $4.1^{*}$ \\
\hline Mesencephalon (substantia nigra) & L & -6 & -15 & -12 & & $3.4^{*}$ \\
\hline \multicolumn{7}{|l|}{ Group by word interaction } \\
\hline Mesencephalon (substantia nigra) & $\mathrm{R}$ & 3 & -15 & -12 & 21 & $3.3^{*}$ \\
\hline \multicolumn{7}{|l|}{ Correlations with word fluency: all subjects ${ }^{\dagger}$} \\
\hline Mesencephalon & $\mathrm{L}$ & -6 & -15 & -18 & 9 & $+2.7^{* *}$ \\
\hline Mesencephalon & $\mathrm{R}$ & 3 & -18 & -21 & & $+2.6^{* *}$ \\
\hline \multicolumn{7}{|l|}{$\begin{array}{l}\text { Mesencephalon correlations with other whole- } \\
\text { brain responses: all subjects }{ }^{\ddagger}\end{array}$} \\
\hline Mesencephalon (substantia nigra): seed value & $\mathrm{R}$ & 3 & -15 & -12 & 250 & +43.9 \\
\hline Cingulum/parahippocampal gyrus (BA 28) & $\mathrm{L}$ & -21 & -24 & -12 & & +3.5 \\
\hline \multicolumn{7}{|l|}{ Medial orbitofrontal cortex, rectal gyrus (BA } \\
\hline 10,11$)$ & $\mathrm{L}$ & -15 & 42 & -9 & 497 & -4.8 \\
\hline Anterior cingulate cortex (BA 24) & B & 0 & 36 & 12 & & -4.0 \\
\hline \multicolumn{7}{|l|}{ Subgenual anterior cingulate cortex (BA 11, } \\
\hline 25) & $\mathrm{R}$ & 3 & 15 & -3 & & -3.7 \\
\hline \multicolumn{7}{|c|}{${ }^{a}$ A positive $t$-stat $(+)$ indicates a positive correlation; a negative $t$-stat $(-)$ indicates a negative correlation. } \\
\hline \multirow{2}{*}{\multicolumn{7}{|c|}{$\begin{array}{l}{ }^{* * * *} \text { For all results, } p<0.05 \text { cluster level corrected (and } p<0.005 \text { voxel level uncorrected), } 4 \text { voxels minimum, }{ }^{*} \text { with a small volume correction (Friston, 1997) applied only to the mesencephalon (**here the voxel level uncorrected threshold } \\
\text { was } p<0.01 ; \text { a small volume correction was also applied). } \\
\text { 'Simple regression analysis between drug }>\text { neutral individual contrast maps and the respective differential word fluency measures as seed values. }\end{array}$}} \\
\hline & & & & & & \\
\hline \multicolumn{7}{|c|}{$\begin{array}{l}\text { FSimple regression analysis between drug }>\text { neutral individual contrast maps and the respective mesencephalon (at } x=3, y=-15, z=-12 \text { ) responses as seed values (correlation with the prefrontal cortical cluster in the controls: } r= \\
-0.7, p<0.01 \text {; in the CUDs: } r=-0.6, p<0.05 \text { ). }\end{array}$} \\
\hline L, Left; R, right; B, bilateral. & & & & & & \\
\hline
\end{tabular}

[note that subtraction between different task conditions, and not just between a task condition and a given baseline, allows for a rigorous control of all processes that are extraneous to the effect of interest (e.g., comparing drug words to neutral words allows for the control of all the sensory-motor properties that are common to these two conditions)]. Brain activation clusters were corrected for multiple comparisons using the continuous random field calculation (Adler, 1981). This approach, as implemented in SPM2, takes into account the local smoothness of the data and is considered more accurate for local smoothness $>3$ voxels than is the more conservative Bonferroni correction (Worsley et al., 1996; Worsley, 2007). In the present study, the random field calculation was based on the expected Euler characteristics of the regions above a $p_{\text {uncorr }}<0.005$ threshold (voxel level uncorrected). Clusters with at least 4 voxels $\left(108 \mathrm{~mm}^{3}\right)$ and $p_{\text {corr }}<0.05$ (cluster level corrected for multiple comparisons) were considered significant. In all SPM analyses, anatomical specificity was corroborated with a coplanar stereotaxic atlas of the human brain (Talairach and Tournoux, 1988).

For followup statistical analyses and to confirm the whole-brain voxelbased analyses, functional regions of interest (ROIs) with an isotropic volume of 27 voxels $\left(729 \mathrm{~mm}^{3}\right)$ were defined at the mesencephalon level (fixed across subjects and conditions) (Table 2) to extract (with a custom program written in IDL, ITT Visual Information Solutions) the average (and variability) BOLD-fMRI signal amplitudes in these coordinates. These ROI measures were used in the appropriate (e.g., $t$ test, correlation) analyses in SPSS 11.5. Statistical significance for these ROI analyses was defined as $p_{\text {uncorr }}<0.05$. These ROI analyses were only performed for the regions that survived the whole-brain correction threshold as described above, which provided protection against type I error.

Finally, all continuous and normally distributed variables were inspected with parametric tests (within groups, paired $t$ test; between groups, independent $t$ tests; correlations, Pearson $r$ ). Otherwise, the respective nonparametric tests were used [Wilcoxon, Mann-Whitney $U$, or Spearman $\left.r\left(r_{\mathrm{s}}\right)\right]$. To study the potential contribution to results of a history of cigarette smoking (that significantly differed between the study groups) (Table 1), differences in all dependent variables between the cigarette smokers and nonsmokers were inspected with $t$ tests or the equivalent nonparametric test; we also inspected correlations between all of our dependent variables and daily frequency of cigarettes smoked (Stevens, 1992). Note that these latter analyses were only performed across all study subjects because within-group analyses would be statistically underpowered (there were only four current or past cigarette smokers within the control group and only three nonsmokers within the CUD group).

\section{Results \\ Drug fluency}

A repeated measures ANOVA with verbal fluency (corrected for verbal intellectual functioning) (supplemental material, available at www.jneurosci.org) revealed a group (CUDs, control) by word (drug, neutral) interaction $\left(F_{(1,28)}=9.0, p<0.01\right)$ (Fig. $\left.1 A\right)$. Post hoc $t$ tests showed that this interaction was explained by higher drug than neutral responses in the CUDs $\left(t_{(14)}=2.2, p<0.05\right)$ but not in healthy participants (who showed a trend toward the reverse pattern, $\left.t_{(14)}=-2.1, p=0.056\right)$ and a significant difference between the study groups for the drug words only (drug: $t_{(28)}$ $=2.2, p<0.05$; neutral: $\left.t_{(28)}=-0.9, p>0.3\right)$.

\section{SPM and ROIs}

Voxel-by-voxel whole-brain analyses (two one-way $t$ tests for the direct drug $>$ neutral comparison as a function of study group) revealed a bilateral mesencephalon response in the CUDs only (Fig. 1, top right, $x=6 /-6, y=-12 /-15, z=-9 /-12,39$ voxels, $t=4.1$ ) (Table 2). Moreover, a direct whole-brain group comparison (two-way $t$ test using the direct drug $>$ neutral contrasts) showed a significant group by word interaction in the right mesencephalon $(x=3, y=-15, z=-12,21$ voxels, $t=3.3)$. The BOLD-fMRI signal (percentage change from baseline) extracted from this region for each participant and used in a repeated measures ANOVA validated this interaction $\left(F_{(1,28)}=\right.$ $10.6, p<0.01$ ) (Fig. $1 B$ ). Post hoc $t$ tests showed that, similarly to the verbal fluency results, this interaction was driven by higher drug than neutral responses in the CUDs $\left(t_{(14)}=3.5, p<0.01\right)$ but not healthy control participants $\left(t_{(14)}=-1.3, p>0.2\right)$. Note that there were no main effects for repetition/block or money on the neural responses to this task (specifically for the direct drug $>$ 
neutral contrast) as further described in supplemental material, available at www.jneurosci.org.

\section{Whole-brain SPM correlations}

Most interestingly, a whole-brain simple regression analysis showed a significant correlation between the differential drug $>$ neutral verbal fluency (used as seed values) and the respective mesencephalon responses across all study participants (observed bilaterally: $x=-6 / 3, y=$ $-15 /-18, z=-18 /-21,9$ voxels, $t=$ 2.7). The BOLD-fMRI signal (percentage change from the direct drug $>$ neutral contrasts extracted from the cluster peak in the left mesencephalon) confirmed that this correlation was driven by the CUDs $(r=0.58, p<0.05)$ and not healthy control participants $(r=0.15, p>0.5)$ (Fig. $1 C)$. The test of coincidence of these regression lines was significant $\left(F_{(2,26)}=3.7\right.$, $p<0.05)$, confirming that these specific brain-behavior correlations differed significantly between the study groups (this result is especially sound given that the whole-brain regression analyses were conducted across all study subjects, enhancing the possibility for uncovering similar group results but decreasing the possibility for differential group results, which were nevertheless significant in the current study). Thus, the more the mesencephalon responded to the drug words compared with the neutral words, the higher was the drug than neutral verbal fluency in the CUDs but not healthy control participants, together attesting to a concomitant brain-behavior drug cue reactivity in cocaine addiction.

Correlations between the mesencephalic drug cue responsivity [seed values were the percentage BOLD signal change for every participant at $x=3, y=-15, z=-12$, or the mesencephalic region that showed a differential drug $>$ neutral BOLD response as a function of study group (Table 2)] and other brain regions that showed similar responses (i.e., the direct drug $>$ neutral contrast maps were used) revealed positive correlations with the parahippocampal gyrus and negative correlations with a cluster that included the medial orbitofrontal cortex/rectal gyrus and rostroventral/pregenual anterior cingulate cortex (Table 2). Thus, in all study subjects, the higher the drug $>$ neutral mesencephalic BOLD response during the drug word fMRI task, the higher the respective response in the parahippocampal gyrus and the lower this relative response in the limbic prefrontal cortex.

\section{Effect of cigarette smoking on all behavioral measures and brain activation results}

There were no significant associations between any of the dependent variables of the study [calculated as absolute (drug or neutral) or differential (drug $>$ neutral) scores for all behavioral measures and ROIs] with history of cigarette smoking $(t<|1.3|$, $p>0.2)$. Also, there were no significant correlations between any of the dependent variables of the study with current cigarette smoking frequency $\left(\mathrm{r}_{\mathrm{s}}<|0.4|, p>0.07\right)$.

\section{Discussion}

Performance on standard (i.e., non-drug-related) neuropsychological tasks is frequently compromised in drug-addicted individuals compared with healthy control subjects (Woicik et al., 2009). In contrast, compared with neutral stimuli (including words), drug stimuli/words can enhance behavioral responses in drug-addicted individuals; although relatively better, these unique drug-related behavioral responses predict disadvantageous treatment outcome in this population (Cox et al., 2006). Our results show, for the first time, that drug words (uniquely human learned verbal descriptors of stimuli) increased fMRIBOLD responses in the mesencephalon, a major source of dopaminergic release to motivationally salient or conditioned stimuli (Robinson and Berridge, 1993; McClure et al., 2003) in cocaineaddicted individuals. These results may reflect the strong conditioned incentive properties of the drug words in the addicted group. It is possible that this increased mesencephalic response to drug words reflects activation from prefrontal glutamatergic projections that regulate dopamine cell firing and drive enhanced dopamine responses to conditioned stimuli (Kalivas, 2004; Wise, 2009). Further, possibly through the mesencephalon's extensive connections with the limbic prefrontal cortex (Devinsky et al., 1995), these cue-reactive neural responses may culminate in drug-biased behaviors (e.g., uncontrollable drug seeking or craving) (Goldstein and Volkow, 2002) in drug-addicted/susceptible individuals. This latter interpretation is consistent with the significant correlations in our study between the drug-related responses in the mesencephalon with similar responses in the prefrontal cortex (Table 2); however, this interpretation remains to be validated with additional direct measures of drug-biased responses in drug-addicted individuals. 
A confound in our study is the use of monetary gain in this task. Here, evidence suggests that rewarding stimuli are directly related to enhanced dopaminergic tone and increased mesencephalic activation (Koch et al., 2008). Indeed, altered fMRI responses to reward in drug users have been reported previously by our group (Goldstein et al., 2007a) and others (Bjork et al., 2008) as indicative of dopaminergic alterations in addiction (Volkow et al., 2004). Nevertheless, in the current study, the word by group interaction in the mesencephalon was observed after averaging the fMRI-BOLD signal across all monetary reward conditions, suggesting that the contribution of drug word cue reactivity in this region in addiction is significant and unique. Another limitation is that results could be attributed to several neuropsychological mechanisms, including enhanced drug word familiarity, salience, attention bias, and memory processes in the CUDs compared with controls. The fMRI task controlled for some of these processes (e.g., the drug and neutral words were matched for frequency in the English language). However, similar processes remain to be tested in the self-generated drug fluency task (e.g., with simultaneous fMRI or recordings of autonomic responses). Finally, the current results need to be replicated in other subgroups of drug-addicted individuals (e.g., treatment seekers recruited from treatment centers and larger samples in which the potential impact on results of individual variables such as sex and race can be studied).

In summary, our results are consistent with the effect of drug words on other (less localized/scalp) psychophysiological responses (Herrmann et al., 2000) and with neuroimaging studies showing similar cue-induced increases in dopaminergic responses when addicted individuals view drug images (pictures or movies) as associated with craving, withdrawal symptoms, and addiction severity (Heinz et al., 2004; Volkow et al., 2006). Our results for the first time demonstrate that, in addicted individuals, drug words alone can elicit an fMRIBOLD mesencephalic response, as possibly associated with dopaminergic (and glutamatergic) mechanisms (Schott et al., 2008) that are crucial to conditioning (D'Ardenne et al., 2008). Moreover, the correlation between a very brief verbal fluency test, which can be easily administered (crucial for clinical studies), and fMRI cue reactivity could be used as a biomarker of neurobiological changes in drug addiction.

\section{References}

Adler RJ (1981) The geometry of random fields. Chichester: Wiley.

Ashburner J, Neelin P, Collins DL, Evans A, Friston K (1997) Incorporating prior knowledge into image registration. Neuroimage 6:344-352.

Beck AT, Steer RA, Brown GK (1996) Beck depression inventory manual, Ed 2. San Antonio, TX: The Psychological Corporation.

Bjork JM, Smith AR, Hommer DW (2008) Striatal sensitivity to reward deliveries and omissions in substance dependent patients. Neuroimage 42:1609-1621.

Bossert JM, Liu SY, Lu L, Shaham Y (2004) A role of ventral tegmental area glutamate in contextual cue-induced relapse to heroin seeking. J Neurosci 24:10726-10730.

Caparelli EC, Tomasi D, Arnold S, Chang L, Ernst T (2003) k-Space based summary motion detection for functional magnetic resonance imaging. Neuroimage 20:1411-1418.

Cox WM, Fadardi JS, Pothos EM (2006) The addiction-stroop test: theoretical considerations and procedural recommendations. Psychol Bull 132:443-476.

D’Ardenne K, McClure SM, Nystrom LE, Cohen JD (2008) BOLD responses reflecting dopaminergic signals in the human ventral tegmental area. Science 319:1264-1267.

Devinsky O, Morrell MJ, Vogt BA (1995) Contributions of anterior cingulate cortex to behaviour. Brain 118:279-306.

First MB, Spitzer RL, Gibbon M, Williams J (1996) Structured clinical in- terview for DSM-IV axis I disorders - patient edition (SCID-I/P, Version 2.0). New York: Biometrics Research Department, New York State Psychiatric Institute.

Francis NW, Kučera H (1982) Frequency analysis of English usage. Boston: Houghton Mifflin.

Friston KJ (1997) Testing for anatomically specified regional effects. Hum Brain Mapp 5:133-136.

Friston KJ, Holmes AP, Worsley KJ, Poline JB, Frith CD, Frackowiak RS (1995) Statistical parametric maps in functional imaging: a general approach. Hum Brain Mapp 2:189-210.

Goldstein RZ, Volkow ND (2002) Drug addiction and its underlying neurobiological basis: neuroimaging evidence for the involvement of the frontal cortex. Am J Psychiatry 159:1642-1652.

Goldstein RZ, Alia-Klein N, Tomasi D, Zhang L, Cottone LA, Maloney T, Telang F, Caparelli EC, Chang L, Ernst T, Samaras D, Squires NK, Volkow ND (2007a) Is decreased prefrontal cortical sensitivity to monetary reward associated with impaired motivation and self-control in cocaine addiction? Am J Psychiatry 164:43-51.

Goldstein RZ, Tomasi D, Rajaram S, Cottone LA, Zhang L, Maloney T, Telang F, Alia-Klein N, Volkow ND (2007b) Role of the anterior cingulate and medial orbitofrontal cortex in processing drug cues in cocaine addiction. Neuroscience 144:1153-1159.

Goldstein RZ, Woicik PA, Lukasik T, Maloney T, Volkow ND (2007c) Drug fluency: a potential marker for cocaine use disorders. Drug Alcohol Depend 89:97-101.

Heinz A, Siessmeier T, Wrase J, Hermann D, Klein S, Grüsser SM, GrüsserSinopoli SM, Flor H, Braus DF, Buchholz HG, Gründer G, Schreckenberger M, Smolka MN, Rösch F, Mann K, Bartenstein P (2004) Correlation between dopamine $\mathrm{D}(2)$ receptors in the ventral striatum and central processing of alcohol cues and craving. Am J Psychiatry 161:1783-1789.

Hennig J, Scheffler K (2001) Hyperechoes. Magn Reson Med 46:6-12.

Herrmann MJ, Weijers HG, Wiesbeck GA, Aranda D, Böning J, Fallgatter AJ (2000) Event-related potentials and cue-reactivity in alcoholism. Alcohol Clin Exp Res 24:1724-1729.

Kalivas PW (2004) Glutamate systems in cocaine addiction. Curr Opin Pharmacol 4:23-29.

Kampman KM, Volpicelli JR, McGinnis DE, Alterman AI, Weinrieb RM, D'Angelo L, Epperson LE (1998) Reliability and validity of the cocaine selective severity assessment. Addict Behav 23:449-461.

Koch K, Schachtzabel C, Wagner G, Reichenbach JR, Sauer H, Schlösser R (2008) The neural correlates of reward-related trial-and-error learning: an fMRI study with a probabilistic learning task. Learn Mem 15:728-732.

Lee JH, Garwood M, Menon R, Adriany G, Andersen P, Truwit CL, Uðurbil K (1995) High contrast and fast three-dimensional magnetic resonance imaging at high fields. Magn Reson Med 34:308-312.

Lezak MD (1995) Neuropsychological assessment, Ed 3. New York: Oxford UP.

McClure SM, Daw ND, Montague PR (2003) A computational substrate for incentive salience. Trends Neurosci 26:423-428.

McLellan AT, Kushner H, Metzger D, Peters R, Smith I, Grissom G, Pettinati $\mathrm{H}$, Argeriou M (1992) The fifth edition of the addiction severity index. J Subst Abuse Treat 9:199-213.

Oldfield RC (1971) The assessment and analysis of handedness: the Edinburgh inventory. Neuropsychologia 9:97-113.

Robinson TE, Berridge KC (1993) The neural basis of drug craving: an incentive-sensitization theory of addiction. Brain Res Brain Res Rev $18: 247-291$.

Schott BH, Minuzzi L, Krebs RM, Elmenhorst D, Lang M, Winz OH, Seidenbecher CI, Coenen HH, Heinze HJ, Zilles K, Düzel E, Bauer A (2008) Mesolimbic functional magnetic resonance imaging activations during reward anticipation correlate with reward-related ventral striatal dopamine release. J Neurosci 28:14311-14319.

Stevens J (1992) Applied multivariate statistics for the social sciences, Ed 2. Hillsdale, NJ: Lawrence Erlbaum Associates.

Talairach J, Tournoux P (1988) Co-planar stereotaxic atlas of the human brain. New York: Thieme Medical.

Tiffany ST, Singleton E, Haertzen CA, Henningfield JE (1993) The development of a cocaine craving questionnaire. Drug Alcohol Depend 34:19-28.

Tomasi D, Caparelli EC, Chang L, Ernst T (2005) fMRI-acoustic noise alters brain activation during working memory tasks. Neuroimage 27:377-386.

Ventura J, Liberman RP, Green MF, Shaner A, Mintz J (1998) Training and 
quality assurance with the Structured Clinical Interview for DSM-IV (SCID-I/P). Psychiatry Res 79:163-173.

Volkow ND, Fowler JS, Wang GJ, Swanson JM (2004) Dopamine in drug abuse and addiction: results from imaging studies and treatment implications. Mol Psychiatry 9:557-569.

Volkow ND, Wang GJ, Telang F, Fowler JS, Logan J, Childress AR, Jayne M, Ma Y, Wong C (2006) Cocaine cues and dopamine in dorsal striatum: mechanism of craving in cocaine addiction. J Neurosci 26:6583-6588.

Wechsler D (1999) Wechsler abbreviated scale of intelligence. San Antonio, TX: The Psychological Corporation.

Whalen PJ, Bush G, McNally RJ, Wilhelm S, McInerney SC, Jenike MA, Rauch SL (1998) The emotional counting Stroop paradigm: a functional magnetic resonance imaging probe of the anterior cingulate affective division. Biol Psychiatry 44:1219-1228.
Wilkinson G (1993) The wide-range achievement test 3 administration manual. Wilmington, DE: Wide Range.

Wise RA (2009) Ventral tegmental glutamate: a role in stress-, cue-, and cocaine-induced reinstatement of cocaine-seeking. Neuropharmacology 56 [Suppl 1]:174-176.

Woicik PA, Moeller SJ, Alia-Klein N, Maloney T, Lukasik TM, Yeliosof O, Wang GJ, Volkow ND, Goldstein RZ (2009) The neuropsychology of cocaine addiction: recent cocaine use masks impairment. Neuropsychopharmacology 34:1112-1122.

Worsley K (2007) Random field theory. In: Statistical parametric mapping: the analysis of functional brain images (Friston K, Ashburner J, Kiebel S, Nichols T, Penny W, eds) pp 232-237. London: Elsevier.

Worsley KJ, Marrett S, Neelin P, Vandal AC, Friston KJ, Evans AC (1996) A unified statistical approach for determining significant signals in images of cerebral activation. Hum Brain Mapp 4:58-73. 